standardised and visualised using the Python Pandas data analysis library and matplotlib.

Results There is evidence of a cohort effect with age-specific IPF death rates increasing in successive cohorts, most clearly seen from age 60. Overall rates were higher for men but there were not marked sex differences in cohort mortality trends (data not shown).

Conclusions The birth cohort effect we observed is consistent with a proportion of IPF cases being due to an occupational or environmental exposure with latency and further research is needed.

\section{Early detection and screening in TB}

\section{P161 TUBERCULOSIS CONTACT SCREENING: WILL THE 2016 GUIDELINES LEAD TO MISSED DIAGNOSES?}

J Prynn, ECJ Bailey, M Darmalingam. Barts Health NHS Trust, London, UK

\subsection{6/thoraxjnl-2017-210983.303}

Background UK TB contact screening guidelines changed in $2016,{ }^{1}$ requiring screening only of contacts of patients with potentially transmissible disease, specifically pulmonary or laryngeal TB. TB contacts and the index case are likely to have had similar TB exposure, and it is possible that contacts who have not been directly infected by the index case will be missed using the new screening guidelines.

Aims and Objectives This study aimed to evaluate whether all cases of TB diagnosed through contact tracing in 2012 at a University Hospital would have been identified using the new screening guidelines.

Methods Case notes of all patients contact screened for TB aged 16 and over in 2012 were examined. Data were collected on the diagnosis of the patient screened (negative, latent, or active TB) and the site of $\mathrm{TB}$ of the index case.

Results Of the 445 screened, $394(88.5 \%)$ were negative for $\mathrm{TB}, 44(9.9 \%)$ had latent $\mathrm{TB}$, and $7(1.6 \%)$ had active $\mathrm{TB}$. For $19.6 \%$ of those with latent or active $\mathrm{TB}$ diagnoses, the $\mathrm{TB}$ site in the index case was neither pulmonary nor laryngeal.

Conclusion By restricting contact screening of this 2012 cohort to those with pulmonary or laryngeal TB contact, 10 (19.6\%) cases of TB would potentially have been missed. As these missed contacts and the TB index case may have had exposure to $\mathrm{TB}$ from the same, albeit unknown, person with a transmissible form of $\mathrm{TB}$, the only way to identify these cases is with a broad screening approach. While the current guidelines are in place, this study highlights the importance of assessing the need for contact screening based on the individual clinical picture in each identified case of TB.

\section{REFERENCE}

1. National Institute of Clinical Excellence. Tuberculosis Guidance: Update Information 2016.

\section{P162 ANALYSIS OF AND LESSONS FROM THE MULTIPLE SCREENING EPISODES IN UK'S TUBERCULOSIS PRE- ENTRY SCREENING PROGRAMME: OCTOBER 2005 TO DECEMBER 2016}

MC Muzyamba, R Harris, D Zenner. Public Health England, London, UK

\subsection{6/thoraxjnl-2017-210983.304}

Background The UK has been carrying out pre-entry tuberculosis (TB) screening for all long term migration visa applicants in 15 pilot countries since October 2005. Screening was subsequently rolled out to visa applicants in 101 high TB incidence $(\geq 40 / 100 \quad 000$ population $)$ countries. Individuals screened free of pulmonary TB are issued with medical clearance certificates valid for 3 and 6 months for those with or without close contact with a TB case, respectively. Consequently, individuals who travelled to the UK on multiple occasions need rescreening once their certificates have expired. Screening identifies prevalent $\mathrm{TB}$ cases among migrants thereby reducing incidence and onward transmission in the UK.

Methods Our study used a cohort of migrants from 40 high incidence countries screened for TB pre-entry by the International Organisation for Migration between October 2005 and December 2016. All applicants screening prior to certificate expiry were excluded. Odds ratios (ORs) and relative risk ratios (RRRs) were estimated using logistic and multinomial logistic regression, respectively, to identify factors associated with multiple screening.

Results The cohort consisted of 855591 migrants screened for pulmonary TB. $729232(85.0 \%)$ were screened once but $126358(15.0 \%)$ were screened $\geq 2$ times. After adjusting for age and sex, the factors associated with being screened $\geq 2$ times included being diagnosed with TB (OR 3.40; 95\% CI 2.99-3.85), close contact with TB (OR 1.29 95\% CI 1.181.40 ) and certain visa types (Settlement: 1.95 ; 95\% CI $1.93-$ 1.97, baseline Student visa). Applicants from the Indian subcontinent were more likely to screen multiple times than other regions. RRRs for individual categories using multinomial logistic regression were similar to overall ORs. Multiple screenings were also associated with increased TB detection. Conclusions Being diagnosed with $\mathrm{TB}$, being a close contact of a TB case and those applying for settlements visa were more likely to be screened multiple times. This information could be used for targeted follow up of individuals likely to screen multiple times and determine their risk of TB.

\section{P163 AN AUDIT INTO THE COMPLETENESS OF LATENT TUBERCULOSIS SCREENING IN THE GASTROINTESTINAL DEPARTMENT, PRIOR TO PATIENTS STARTING ANTI- TNF-A THERAPY}

SM Meghji. University of Southampton, Southampton, UK

10.1136/thoraxjnl-2017-210983.305 